\title{
Correction to: Testing creation cold dark matter cosmology with the radiation temperature-redshift relation
}

\author{
luri P. R. Baranov ${ }^{1,2,3}$. José F. Jesus ${ }^{4,5}$. José A. S. Lima ${ }^{1}$
}

Published online: 12 November 2020

๑) Springer Science+Business Media, LLC, part of Springer Nature 2020

\section{Correction to: General Relativity and Gravitation General Relativity and Gravitation (2019) 51:33 https://doi.org/10.1007/s10714-019-2516-3}

There is a typo in Equation (2) of the article. It should be written as:

$$
8 \pi G \sum_{i=1}^{N}\left(p_{i}+P_{c i}\right)=-2 \dot{H}-3 H^{2},
$$

It has no consequences on the results of the paper.

Publisher's Note Springer Nature remains neutral with regard to jurisdictional claims in published maps and institutional affiliations.

The original article can be found online at https://doi.org/10.1007/s10714-019-2516-3.

$凶 \quad$ Iuri P. R. Baranov

iuribaranov@gmail.com

José F. Jesus

jfjesus@itapeva.unesp.br

José A. S. Lima

jas.lima@iag.usp.br

1 Departamento de Astronomia, Universidade de São Paulo, R. do Matão 1226, São Paulo, SP 05508-900, Brazil

2 Instituto Federal do Paraná, R. Felipe Tequinha 1400, Paranavaí, PR 87703-536, Brazil

3 Instituto Federal de Educação, Ciência e Tecnologia da Bahia, Campus Simões Filho, Via Universitária s/n, Pitanguinha, Simões Filho, BA 43700-000, Brazil

4 Universidade Estadual Paulista (Unesp), Câmpus Experimental de Itapeva, R. Geraldo Alckmin 519, Itapeva, SP 18409-010, Brazil

5 Faculdade de Engenharia, Guaratinguetá, Universidade Estadual Paulista (Unesp), Av. Ariberto Pereira da Cunha 333, Guaratinguetá, SP 12516-410, Brazil 